Abstract

\title{
Entropy of Vostok Ice Core Data and Kalman Filter Harmonic Bank Climate Predictor ${ }^{\dagger}$
}

\author{
Migdat Hodzic ${ }^{1}$ and Ivan Kennedy ${ }^{2}$ \\ 1 AUBIH \\ 2 University of Sydney, Sydney, Australia \\ + Presented at the Entropy 2021: The Scientific Tool of the 21st Century, 5-7 May 2021; Available online: \\ https://sciforum.net/conference/Entropy2021/.
}

Published: 5 May 2021

Entropy of Vostok ice core data together with our notion of Kalman Filter Harmonic Bank (KFHB) Climate Prediction Engine (CPE) are introduced in this paper. In particular we examine $\mathrm{CO}_{2} \mathrm{Cycle} 1$ data (the most recent data cycle), and analyze so called Spectral Entropy of $\mathrm{CO}_{2}$ harmonics obtained by standard Fast Fourier Transform (FFT) analysis. We also introduce treatment of Vostok Data as a sample from a corresponding non stationary stochastic process for which, instead of FFT, we can use Karhunen-Loeve Expansion (KLE) for a set of discrete data values and the corresponding autocorrelation matrix, defining Representation Entropy as a broader concept compared to Spectral Entropy for FFT. Initial results for Spectral Entropy are presented as a measure of amplitude and energy analysis informational effectiveness which determines a set of signal harmonics implemented in a form of KFHB whereas each harmonic is generated by a two state Kalman Filter. The total signal is then represented as a sum of a set of amplitude or energy significant harmonics (hence the name Kalman Filter Harmonic Bank). Spectral Entropy calculations point to a suitable number of FFT generated harmonics to be used for signal synthesis by harmonic truncation. We also analyze using amplitude vs. energy (amplitude squared) as a base for entropic calculations. Similarly in the case of KLE, Representation Entropy would play the same role. Ultimately we are working to implement this approach into an effective Machine Learning short and long term CPE. It is critical to perform very detailed time and frequency data analysis as a solid base for the CPE methodology for modelling variations in climate.

(C) 2021 by the authors. Licensee MDPI, Basel, Switzerland. This article is an open access article distributed under the terms and conditions of the Creative Commons Attribution (CC BY) license (http://creativecommons.org/licenses/by/4.0/). 